OPEN ACCESS

Edited by:

Eiman Aleem,

The University of Arizona College of

Medicine - Phoenix, United States

Reviewed by:

Yibo Luo,

The University of Toledo,

United States

Pietro Gareri,

Center for Cognitive Disorders and

Dementia, Asp Catanzaro, Italy

Pawel Grieb,

Mossakowski Medical Research Centre, Polish Academy of Sciences,

Poland

*Correspondence.

Yilai Shu

yilai_shu@fudan.edu.cn

Renjie Chai

renjiec@seu.edu.cn

Xia Gao

xiagaogao@hotmail.com

†These authors have contributed equally to this work

Specialty section:

This article was submitted to Cell Growth and Division,

a section of the journal

Frontiers in Cell and Developmental

Biology

Received: 02 April 2020

Accepted: 13 July 2020

Published: 31 August 2020

Citation:

Zhong Z, Fu X, Li H, Chen J, Wang $M$, Gao S, Zhang L, Cheng $C$, Zhang Y, Li P, Zhang S, Qian X, Shu Y,

Chai $R$ and Gao X (2020) Citicoline Protects Auditory Hair Cells Against

Neomycin-Induced Damage.

Front. Cell Dev. Biol. 8:712.

doi: 10.3389/fcell.2020.00712

\section{Citicoline Protects Auditory Hair Cells Against Neomycin-Induced Damage}

\author{
Zhenhua Zhong ${ }^{1,2+}$, Xiaolong $\mathrm{Fu}^{3 t}$, He $\mathrm{Li}^{4 t}$, Jie Chen ${ }^{1+}$, Maohua Wang ${ }^{5}$, Song $\mathrm{Gao}^{6}$, \\ Liyan Zhang ${ }^{3}$, Cheng Cheng', Yuan Zhang ${ }^{3}$, Peipei Li i', Shasha Zhang ${ }^{3}$, Xiaoyun Qian', \\ Yilai Shu ${ }^{8 *}$, Renjie Chai ${ }^{3,9,10,11 *}$ and Xia Gao ${ }^{1 *}$
}

\begin{abstract}
1 Jiangsu Provincial Key Medical Discipline (Laboratory), Department of Otolaryngology Head and Neck Surgery, Nanjing Drum Tower Hospital Clinical College of Nanjing Medical University, Nanjing, China, ${ }^{2}$ Department of Otolaryngology, Head and Neck Surgery, The Affiliated Hospital of Yangzhou University, Yangzhou University, Yangzhou, China, ${ }^{3}$ MOE Key Laboratory for Developmental Genes and Human Disease, Jiangsu Province High-Tech Key Laboratory for Bio-Medical Research, Institute of Life Sciences, Southeast University, Nanjing, China, ${ }^{4}$ Department of Otolaryngology-Head and Neck Surgery, First Affiliated Hospital of Wenzhou Medical University, Wenzhou, China, ${ }^{5}$ Department of Otolaryngology, Head and Neck Surgery, Xiangya School of Medicine, Central South University, Changsha, China, ${ }^{6}$ Department of Otolaryngology, Affiliated People's Hospital of Jiangsu University, Zhenjiang, China, ${ }^{7}$ School of Life Sciences, Shandong University, Jinan, China, ${ }^{8}$ ENT Institute and Department of Otorhinolaryngology, Eye \& ENT Hospital, State Key Laboratory of Medical Neurobiology, Institute of Biomedical Sciences, NHC Key Laboratory of Hearing Medicine, Fudan University, Shanghai, China, ${ }^{9}$ Co-innovation Center of Neuroregeneration, Nantong University, Nantong, China, ${ }^{10}$ Institute for Stem Cell and Regeneration, Chinese Academy of Sciences, Beijing, China, ${ }^{11}$ Beijing Key Laboratory of Neural Regeneration and Repair, Capital Medical University, Beijing, China
\end{abstract}

Aminoglycoside-induced hair cell $(\mathrm{HC})$ loss is one of the most important causes of hearing loss. After entering the inner ear, aminoglycosides induce the production of high levels of reactive oxygen species (ROS) that subsequently activate apoptosis in HCs. Citicoline, a nucleoside derivative, plays a therapeutic role in central nervous system injury and in neurodegenerative disease models, including addictive disorders, stroke, head trauma, and cognitive impairment in the elderly, and has been widely used in the clinic as an FDA approved drug. However, its effect on auditory HCs remains unknown. Here, we used HC-like HEl-OC-1 cells and whole organ explant cultured mouse cochleae to explore the effect of citicoline on aminoglycoside-induced $\mathrm{HC}$ damage. Consistent with previous reports, both ROS levels and apoptosis were significantly increased in neomycin-induced cochlear HCs and HEl-OC-1 cells compared to undamaged controls. Interestingly, we found that co-treatment with citicoline significantly protected against neomycin-induced $\mathrm{HC}$ loss in both HEl-OC1 cells and whole organ explant cultured cochleae. Furthermore, we demonstrated that citicoline could significantly reduce neomycin-induced mitochondrial dysfunction and inhibit neomycin-induced ROS accumulation and subsequent apoptosis. Thus, we conclude that citicoline can protect against neomycin-induced $\mathrm{HC}$ loss by inhibiting ROS aggregation and thus preventing apoptosis in $\mathrm{HCs}$, and this suggests that citicoline might serve as a potential therapeutic drug in the clinic to protect HCs.

\footnotetext{
Keywords: citicoline, hair cell, apoptosis, reactive oxygen species, aminoglycosides
} 


\section{INTRODUCTION}

Sensorineural hearing loss remains a serious sensory disorder worldwide. Once the hair cells (HCs) of the inner ear are damaged, sensorineural hearing loss is permanent due to the inability of mammalian HCs to regenerate. The causes of hearing loss include many factors, such as noise, age, and ototoxic drugs, all of which can induce apoptosis in HCs (Prasad and Bondy, 2020). Damage caused by aminoglycosides, which are the most commonly used ototoxic drugs, is a major cause of HC death (Waguespack and Ricci, 2005). Therefore, it is important to investigate the molecular mechanism behind aminoglycoside-induced auditory sensory cell damage and to seek effective drugs for preventing and treating aminoglycoside-induced deafness. Several studies have suggested that aminoglycosides induce intrinsic apoptosis of HCs through oxidative stress (Mangiardi et al., 2004; Coffin et al., 2013; Sun et al., 2014, 2015; Liu et al., 2016), while others have reported that the accumulation of reactive oxygen species (ROS) plays an important role in the death of HCs (Clerici et al., 1996; Choung et al., 2009). ROS can be cleared by physiological cellular processes; however, they are harmful when their concentration exceeds the cell's capacity to remove them (He et al., 2020). Thus, the survival of HCs requires a balance between oxidative stress and anti-oxidation (Franco and Cidlowski, 2009; Filomeni et al., 2015), suggesting that preventing the accumulation of ROS might be a potential treatment for preventing ototoxicity.

Citicoline, a nucleoside derivative, is an essential endogenous intermediate in phosphatidylcholine biosynthetic pathways. Citicoline is widely distributed in the body, and it can easily cross the blood-brain barrier and penetrate brain cells to provide neural protection (Secades and Lorenzo, 2006). It has been widely demonstrated that citicoline can activate the biosynthesis of phospholipids in neuronal membranes, and this stimulates brain metabolism and the neurotransmitter system (Secades and Lorenzo, 2006). It also plays an important role in promoting the recovery of brain function and in awakening from sleep (Adibhatla et al., 2002). When used as a long-term medication, citicoline is a safe and well-tolerated drug without significant systemic side effects. Citicoline can be used to treat addictive disorders (Wignall and Brown, 2014), stroke, head trauma (Saver, 2008), and cognitive impairment in the elderly (Cotroneo et al., 2013), and it is also effective in the treatment of glaucoma (Parisi et al., 2008). In addition, citicoline has anti-apoptotic effects by disrupting mitochondria-dependent cell death mechanisms and promoting the regeneration of nerve axons, and it can protect retinal ganglion cells from damage (Oshitari et al., 2002).

There have been no reports on whether citicoline can protect auditory HCs from aminoglycoside-induced injury up to now. In our study, we used the HC-like House Ear Institute Organ of Corti 1 (HEI-OC-1) cell line along with explant cultured cochlear HCs to establish an in vitro neomycin-induced damage model in auditory HCs with the aim to investigate the potential protective effect of citicoline in auditory HCs.

\section{MATERIALS AND METHODS}

\section{Animals}

All animal procedures were performed according to protocols approved by the Animal Care and Use Committee of Southeast University, and all efforts were made to minimize the number of animals used and to prevent their suffering.

\section{Cell Cultures and Tissue Cultures}

Consistent with previous studies, we used HEI-OC1 (House Ear Institute-organ of Corti 1) cells derived from long-term cultures of Immortomouse cochleae. HEI-OC1 cells express Atoh1, Prestin, Myo7a, and other cellular markers specific for auditory sensory HCs when cultured either under permissive or non-permissive conditions. The cells were cultured in DMEM containing $10 \%$ fetal bovine serum and $100 \mathrm{IU} / \mathrm{ml}$ penicillin (A0166, Sigma-Aldrich, St. Louis, United States) at $37^{\circ} \mathrm{C}$ and $5 \% \mathrm{CO}_{2}$ (Kalinec et al., 2003; He et al., 2020) and sub-cultured at $80 \%$ confluency using $0.25 \%$ trypsin/EDTA (25200056, Life Technologies, Waltham, MA, United States). Neomycin (N6386, Sigma-Aldrich) was used at a final concentration of $10 \mathrm{mM}$ to damage the HEI-OC-1 cells. Citicoline (C0256, SigmaAldrich) was used at a final concentration of $10 \mu \mathrm{M}$ to treat the HEI-OC-1 cells.

Cochleae were dissected from postnatal day $(\mathrm{P}) 3$ mice and cultured as previously reported (Chen et al., 2013), The explant cultured tissue was pretreated with $10 \mu \mathrm{M}$ citicoline for $12 \mathrm{~h}$, then $0.5 \mathrm{mM}$ neomycin was added for $12 \mathrm{~h}$ to damage the HCs. After removal of the neomycin, the tissues were recovered in serum-free medium for an additional $12 \mathrm{~h}$ together with $10 \mu \mathrm{M}$ citicoline. Animal experiments were conducted in accordance with the guidelines of the Committee of Animal Protection and Utilization of Southeast University and were approved by the Animal Experimental Ethics Committee of Southeast University.

\section{Real-Time PCR}

Total RNA was extracted from HEI-OC-1 cells or whole cochleae with Trizol reagent (PR910, Protein Biotechnology, Beijing, China), and the integrity of the RNA samples was evaluated by OD260/280 measurements. cDNA was obtained using the Revertaid First Strand cDNA Synthesis Kit (K1622, Thermo Fisher Scientific) according to the manufacturer's protocol. Realtime PCR was performed on a Biosystems CFX96 real-time PCR system (Bio-Rad, Hercules, CA, United States) using SYBR Green qRT-PCR Master Mix (4913850001, Roche Life Science, Basel, Switzerland). The primer sequences are listed in Table 1. The qRT-PCR conditions were as follows: initial denaturing for $15 \mathrm{~s}$ at $95^{\circ} \mathrm{C}$ followed by 40 cycles of denaturation at $95^{\circ} \mathrm{C}$ for $15 \mathrm{~s}$, annealing at $60^{\circ} \mathrm{C}$ for $60 \mathrm{~s}$, and extension at $72^{\circ} \mathrm{C}$ for $20 \mathrm{~s}$. The mRNA expression values of the genes of interest were normalized to the mRNA expression of Gapdh. The results were calculated using the comparative cycle threshold $(\Delta \Delta \mathrm{Ct})$ method. 
TABLE 1| PCR sequences used in the experiments.

\begin{tabular}{lll}
\hline Gene & Forward sequence & Reverse sequence \\
\hline Caspase-3 & AATCATGCCATTGCCCAGC & CTCAAGTGTGTAGGGGGAGG \\
Caspase-8 & AGCCTATGCCACCTAGTGAT & GGAGAGCTGTAACCTGTCGC \\
Bcl-2 & GGTGAACTGGGGGAGGATTG & AGAGCGATGTTGTCCACCAG \\
Caspase-9 & CCTAGTGAGCGAGCTGCAAG & ACCGCTTGCAAGAGTGAAG \\
Bax & TGAAGACAGGGGCCTITTG & AATTCGCCGGAGACACTCG \\
Alox15 & TCGGGACTCGGAAGCAGAAT & CCCATCGGTAACAGGGGAAC \\
Gsr & TGCACTTCCCGGTAGGAAAC & GATCGCAACTGGGGTGAGAA \\
Sod1 & GGAGCAAGGTCGCTTACAGA & AGTGACAGCGTCCAAGCAAT \\
Glrx & AGTCTGGAAAGGTGGTCGTG & CCATTAGCATGGCTGGACGA \\
GADPH & GCAAGAGAGAGGCCCTCAG & TGTGAGGGAGATGCTCAGTG \\
\hline
\end{tabular}

\section{Cell Number Analysis}

HEI-OC-1 cells were incubated in 96-well plates for $24 \mathrm{~h}$ at a concentration of 2,000 cells/well in three replicates, and different drugs were added (controls received a similar volume of DMEM). The Cell Counting Kit (CCK-8; CC201, Protein Biotechnology, Beijing, China) was used to determine the cell viability at different time points after incubation or at different concentrations. After exposing the cochleae to citicoline and/or neomycin, the immunostained cells were quantified per $100 \mu \mathrm{m}$ in all three turns of the cochlea. The numbers of positive cells were counted in equal lengths from the apical to the basal turns of the cochlea.

\section{Western Blot}

The HEI-OC-1 cell line and cochlear tissue were lysed with ice-cold RIPA lysis buffer (PP109, Protein Biotechnology) plus Phosphatase Inhibitor Cocktails (04693132001, Roche) for $30 \mathrm{~min}$ at $4^{\circ} \mathrm{C}$. Protein concentrations were determined using a BCA Protein Quantification Kit (PP202, Protein Biotechnology) according to the manufacturer's instructions. Equal amounts of protein were loaded onto a $12 \%$ Tris-glycine SDS-PAGE gel, separated at 120 volts for 1.5 to $2 \mathrm{~h}$, and then transferred to a nitrocellulose membrane and blocked with $5 \%$ milk in PBST [ $1 \times$ PBS with 0.1\% Triton X-100 (Solarbio, 1109F0521)] buffer. Cleaved caspase 3 was evaluated using anti-cleaved caspase 3 rabbit monoclonal antibody (1:1,000 dilution, 9664S, Cell Signaling Technology), and $\beta$-actin was measured using a mouse monoclonal antibody (1:5,000 dilution, ab119716, Abcam, Cambridge, United Kingdom). Peroxidase-conjugated goat antirabbit or anti-mouse immunoglobulin G (ab6789 and ab6721, Abcam) was used as the secondary antibody. The proteins were detected using a SuperSignal West Dura chemiluminescent substrate kit (34075, Thermo Scientific) according to the manufacturer's instructions. Semi-quantification of the western blot results was done by measuring the intensities of the bands using ImageJ.

\section{Immunofluorescence}

Anti-cleaved caspase 3 antibody (1:400 dilution, 9664S, Cell Signaling Technology), MitoSOX Red (M36008, Life Technologies), tetramethylrhodamine ethyl ester perchlorate
(TMRE, Sigma-Aldrich), anti-Myo7A antibody (1:1,000 dilution, 25-6790, Proteus Bioscience), anti-voltage dependent anion channel1 (VDAC1) polyclonal antibody (1:200 dilution, 10866-1-AP, Proteintec), and DAPI (1:1,000 dilution, C0060, Solarbio) were used to analyze apoptotic cells, measure ROS, stain HCs, measure mitochondrial number, and stain nuclei, respectively. Briefly, cells and cochlear tissues were fixed in $4 \%$ paraformaldehyde (158127, Sigma-Aldrich) for $1 \mathrm{~h}$ then washed three times with PBST $[1 \times$ PBS with $0.1 \%$ Triton X-100 (Solarbio, 1109F0521)] and incubated for $1 \mathrm{~h}$ in blocking medium (PBS with 10\% heat-inactivated donkey serum, 1\% Triton $\mathrm{X}-100,1 \% \mathrm{BSA}$, and $0.02 \%$ sodium azide at $\mathrm{pH} 7.2$ ) at room temperature. The samples were marked with primary antibody diluted in PBT-1 (PBS with 10\% Triton X-100, 5\% heat-inactivated donkey serum, $1 \%$ BSA, and $0.02 \%$ sodium azide at $\mathrm{pH}$ 7.2) for overnight at $4^{\circ} \mathrm{C}$. After washing three times with PBST, the samples were marked with the secondary antibody diluted in PBT-2 (PBS with 1\% BSA and 0.1\% Triton X-100 at $\mathrm{pH}$ 7.2) for $1 \mathrm{~h}$. The samples were washed again three times and were imaged by confocal fluorescence microscopy (Leica SP5, Heidelberg, Germany).

The TUNEL Kit (11684817910, Roche, Indianapolis, IN, United States) was used to detect apoptotic cells following the manufacturer's instructions. TMRE was used for measuring the mitochondrial membrane potential (MMP), and Mito-SOX Red was used to analyze ROS levels. Briefly, the culture medium was removed from the dish and the samples were washed with PBS. The samples were then cultured in DMEM containing Mito-SOX Red or TMRE at $37^{\circ} \mathrm{C}$ for $30 \mathrm{~min}$. The samples were then washed in pre-warmed PBS and imaged with a confocal microscope (LSM700; Zeiss, Heidenheim, Germany).

\section{Flow Cytometry}

Annexin V-FITC and propidium iodide (C1062, Beyotime) were used for apoptosis analysis following the manufacturer's instructions. After treating the HEI-OC-1 cells with citicoline and/or drugs, the cells were trypsinized and collected and then washed twice with PBS and resuspended in binding buffer at a concentration of $1 \times 10^{6}$ cells $/ \mathrm{ml}$. Annexin V-FITC and propidium iodide were added and gently mixed with the cells and incubated at room temperature for 10-20 min in the dark. Cells were analyzed as quickly as possible by flow cytometry (FACSCanto, BD, San Jose, CA, United States).

Mito-SOX Red and TMRE were used to analyze ROS production and to measure the MMP, respectively. After treating the HEI-OC-1 cells with citicoline and/or neomycin, the cells were trypsinized, collected, and resuspended in prewarmed solution containing Mito-SOX Red or TMRE for 10 min. Following this, the cells were washed with PBS and analyzed by flow cytometry. All experiments were repeated at least three times.

\section{Statistical Analysis}

All data are shown as mean \pm standard deviation (SD), and all experiments were repeated at least three times. Statistical analyses were performed using Microsoft Excel and GraphPad Prism 6 software (La Jolla, CA, United States). For the cochlear tissue 
culture experiments, " $n$ " represents the number of independent cochleae, and for all HEI-OC-1 cell culture experiments " $n$ " represents the number of replicates. When comparing two groups, a two-tailed, unpaired Student's $t$-test was used to determine statistical significance. When comparing more than two groups, one-way ANOVA was used followed by Dunnett's multiple comparison test. A $p$-value $<0.05$ was considered to be statistically significant.

\section{RESULTS}

\section{The Survival of HEI-OC-1 Cells Is Affected by Neomycin and Citicoline Treatment}

To select the proper conditions to induce cell death in HEI-OC-1 cells, we exposed HEI-OC-1 cells to different doses of neomycin (1-20 mM) for different times $(0-24 \mathrm{~h})$. We found that the viability of HEI-OC-1 cells decreased gradually with increasing neomycin doses and time, and 50-60\% of the HEI-OC-1 cells were alive after being treated with $10 \mathrm{mM}$ neomycin for $24 \mathrm{~h}$. Thus we chose this condition to induce HEI-OC-1 cell damage (Supplementary Figures S1A,B). Because there are no reports of using citicoline to protect against ototoxic drug-induced HC loss, we first determined the appropriate dose and treatment time of citicoline in HEI-OC-1 cells before neomycin exposure. We pre-treated the HEI-OC-1 cells with different concentrations of citicoline $(1,10,100 \mu \mathrm{M}, 1 \mathrm{mM}$, and $2 \mathrm{mM})$ for different times (6, 12 , and $24 \mathrm{~h}$, respectively) and then treated the cells with $10 \mathrm{mM}$ neomycin together with citicoline (the same concentration as pre-treatment) for $24 \mathrm{~h}$. The CCK8 results showed that there was no protective effect of citicoline when added $6 \mathrm{~h}$ in advance, but there was a clear protective effect when citicoline was added 12 or $24 \mathrm{~h}$ in advance. Additionally, the viability of HEI-OC-1 cells gradually increased with low concentrations of citicoline, but once the concentration of citicoline was higher than $10 \mu \mathrm{M}$ the viability of HEI-OC-1 cells began to decrease. We also found that there was no significant difference in cell viability with the different pretreatment times (12 and $24 \mathrm{~h}$ ). Therefore, we chose $10 \mu \mathrm{M}$ citicoline pretreatment for $12 \mathrm{~h}$ as the optimal treatment condition in this study (Supplementary Figures S1C-F).

\section{Citicoline Treatment Protects HCs in Whole Organ Cultured Cochleae Against Neomycin Injury}

Cochleae from P3 mice were used to investigate the role of citicoline in cochlear HCs after neomycin treatment (Figure 1A). We first used Myo7A and DAPI staining to observe the changes in the numbers of HCs in the apical, middle, and basal turns of the cochlea after different treatments. We found that the number of Myo7A and DAPI double-positive cells per $100 \mu \mathrm{m}$ of cochlear length of the apical, middle, and basal turns was significantly lower in the neomycin-only group than in the undamaged controls (Figures 1B,C). In contrast, the number of double-positive cells was significantly increased in the citicoline-treated group compared with the neomycin-only group (Figures 1B,C).

\section{Citicoline Reduces Apoptosis in Cochlear HCs After Neomycin Exposure}

Next, we explored the role of citicoline in neomycin-induced HC injury. Previous studies have shown that cleaved caspase 3 and TUNEL can be used as markers for apoptosis induced by aminoglycosides (Matsui et al., 2002; Coffin et al., 2013; He et al., 2014). Therefore, immunofluorescence staining was used to evaluate the expression of cleaved caspase 3 and TUNEL in cochlear HCs after citicoline pretreatment. The results showed that the numbers of cleaved caspase 3-positive cells and TUNEL-positive cells per $100 \mathrm{~mm}$ of the cochlea in the middle turn were significantly increased in the neomycin-treated group compared with the undamaged controls (Figures 2A-D). Moreover, the citicoline-pretreated cochleae showed significantly lower numbers of caspase 3-positive cells and TUNEL-positive cells than the neomycin-only group (Figures 2A-D). Western blot results also showed that the expression levels of cleaved caspase 3 in the neomycin-only group were higher than in the undamaged controls (Figures 2E,F), while they were significantly decreased in the citicoline-treated group compared with the neomycin-only group (Figures 2E,F).

We also performed quantitative real-time polymerase chain reaction (qRT-PCR) to investigate the expression of apoptosisrelated genes in the cochlea after citicoline treatment. Compared with the undamaged controls, the expression of the intrinsic and extrinsic pro-apoptotic genes Casp3, Casp8, and Casp9 was significantly increased in the neomycin-only group, while the expression of the anti-apoptotic gene $B c l 2$ was significantly decreased and expression of the pro-apoptotic gene Bax was not significantly different (Figure 2G). Notably, citicoline treatment significantly downregulated the expression of the pro-apoptotic genes Bax, Casp3, Casp8, and Casp9 after neomycin exposure (Figure 2G). Together, these results suggest that citicoline reduces apoptosis in cochlear HCs after neomycin exposure.

\section{Citicoline Reduces Apoptosis in HEI-OC-1 Cells After Neomycin Exposure}

To investigate the role of citicoline in neomycin-induced death in HEI-OC-1 cells, the cells were pretreated with $10 \mu \mathrm{M}$ citicoline for $12 \mathrm{~h}$ and then treated with $10 \mathrm{mM}$ neomycin along with citicoline for $24 \mathrm{~h}$ and allowed to recover in culture medium for another $12 \mathrm{~h}$ together with citicoline (Figure 3A). We labeled the dead cells with propidium iodide and labeled the cells undergoing apoptosis with Annexin V. The percentage of apoptotic cells was significantly higher after neomycin treatment compared to the undamaged group, while the percentage was significantly reduced in the citicoline-treated group compared with the neomycin-only group (Figures $3 \mathbf{B}, \mathbf{C}$ ). To verify this result, we performed TUNEL staining to further detect apoptosis in HEI-OC-1 cells. TUNEL staining showed that the proportion of TUNEL-positive cells in the neomycin-induced group was significantly higher than in the undamaged group, while the citicoline-treated group showed significantly lower percentages of 
A

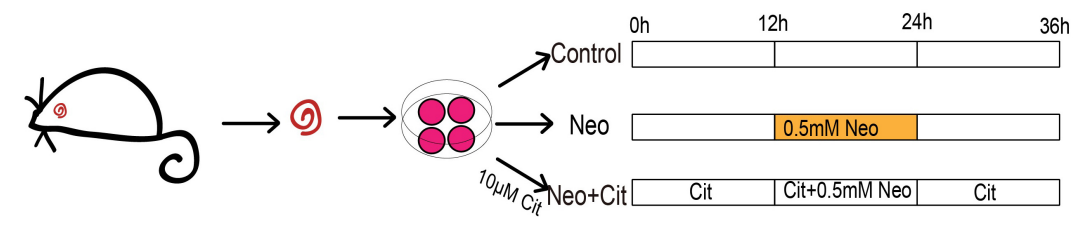

B

Apex

Mid

Base
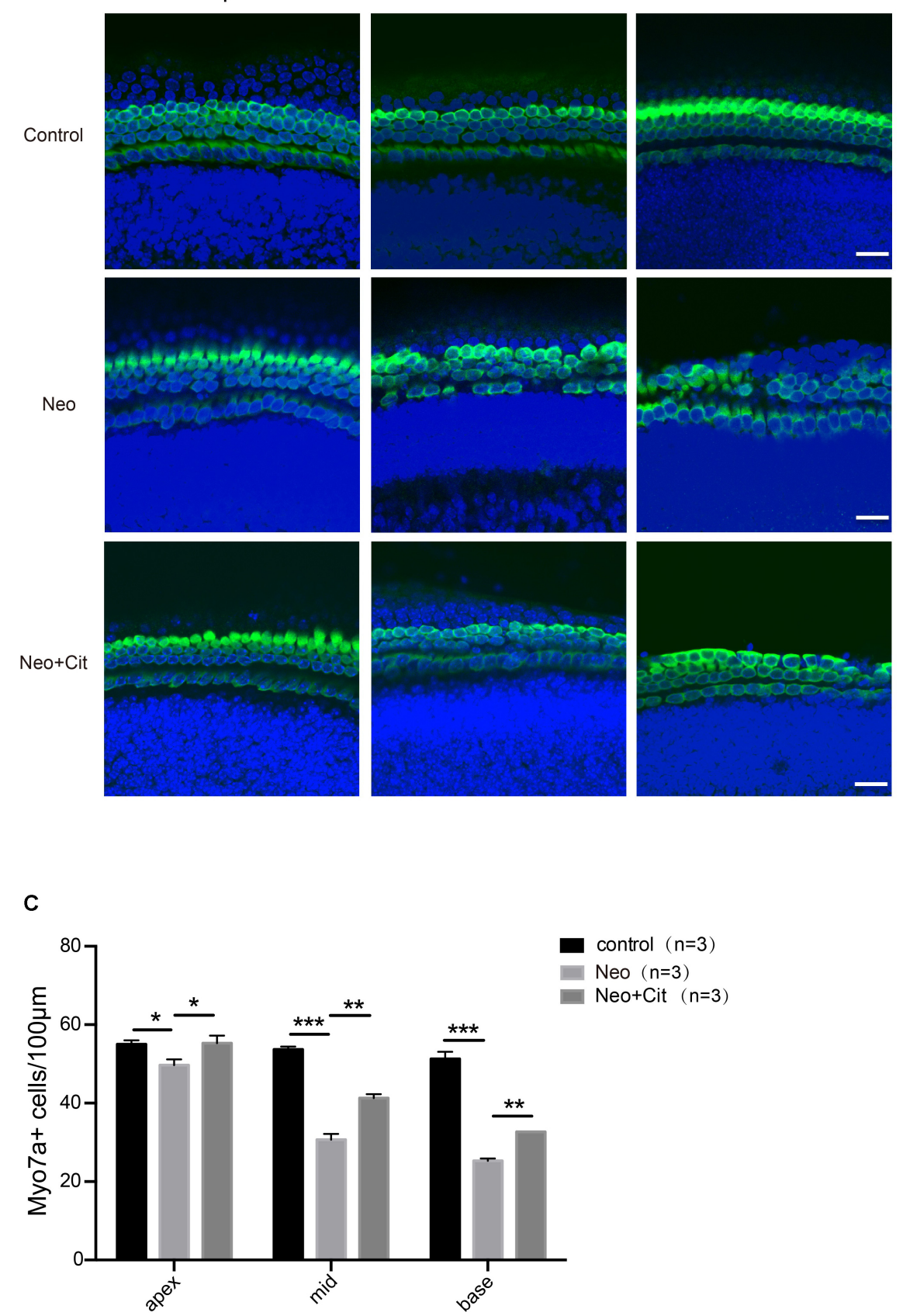

FIGURE 1 | Citicoline protects against apoptosis in cochlear HCs after neomycin injury. (A) Schematic diagram of drug addition in tissue culture.

(B) Immunofluorescence staining with Myo7A (green) and DAPI (blue) in the apical, middle, and basal turns of the cochlear explant cultures with different treatments. (C) Quantification of the numbers of Myo7A and DAPI double-positive cells in (B). Data are shown as mean \pm SD. ${ }^{*} p<0.05,{ }^{* *} p<0.01,{ }^{* \star *} p<0.001$. Scale bars $=20 \mu \mathrm{m}$. 

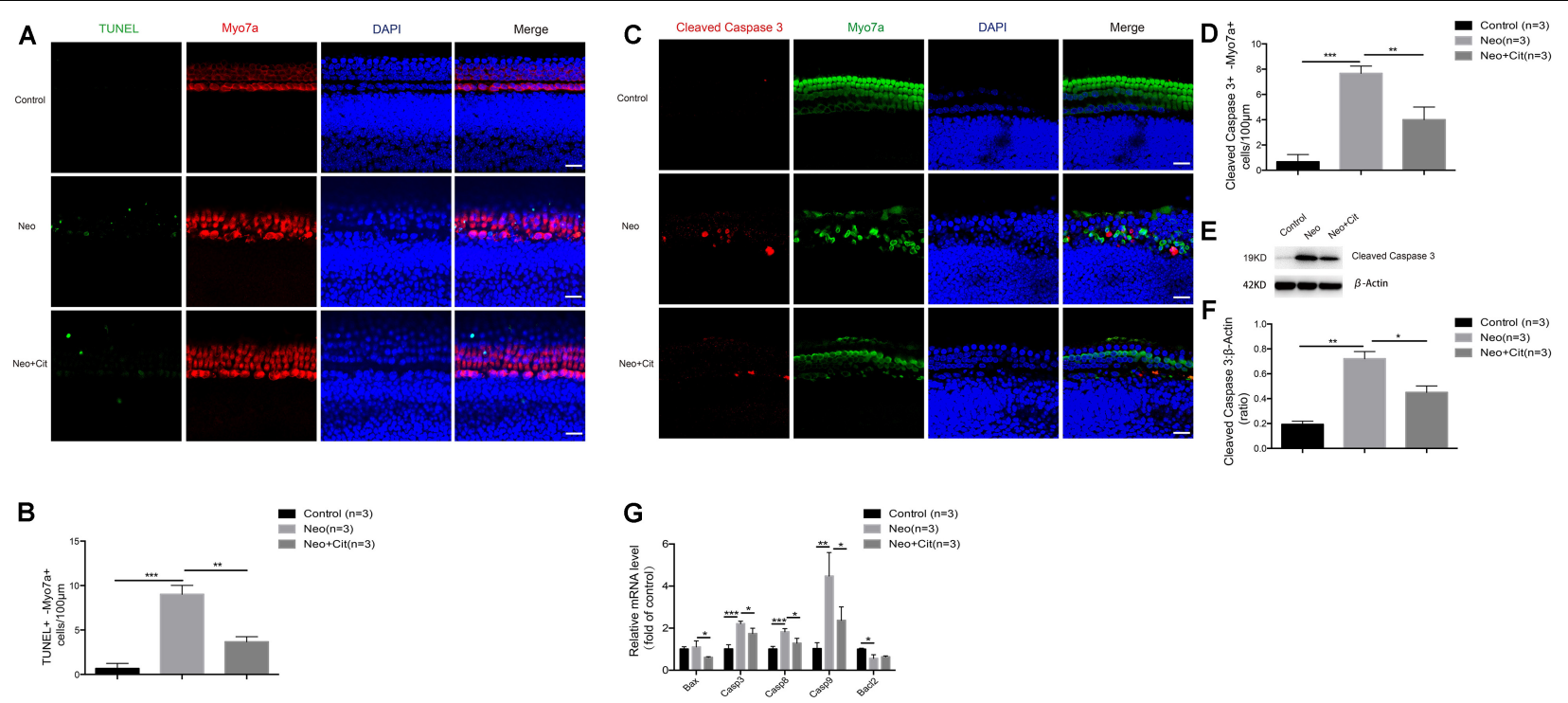

FIGURE 2 | Citicoline reduces the expression of apoptotic factors in cochlear HCs after neomycin exposure. (A) Immunofluorescence staining with TUNEL and Myo7A in the middle turn of the cochlea after different treatments. (B) Quantification of the numbers of TUNEL and Myo7A double-positive cells in (A).

(C) Immunofluorescence staining of cleaved caspase 3 and Myo7A in the middle turn of the cochlea after different treatments. (D) Quantification of the numbers of cleaved caspase 3 and Myo7A double-positive cells in (C). (E) Western blot showing that the amount of cleaved caspase 3 in the neomycin-only group was higher than in the undamaged control. The amount of cleaved caspase 3 induced by neomycin was significantly reduced by pretreatment with citicoline. (F) Quantification of the western blot in (E). (G) The mRNA levels of five apoptosis-related genes were analyzed by qRT-PCR after neomycin and citicoline treatment normalized to GAPDH and presented as the fold change compared to control levels. Data are shown as mean $\pm \mathrm{SD}$. ${ }^{\star} p<0.05,{ }^{\star *} p<0.01,{ }^{\star \star *} p<0.001$. Scale bars $=20 \mu \mathrm{m}$.

TUNEL-positive cells compared with the neomycin-only group (Figures 3D,E). Together, these results show that citicoline decreases apoptosis in HEI-OC-1 cells after neomycin exposure.

\section{Citicoline Reduces the Expression of Apoptotic Factors in HEI-OC-1 Cells After Neomycin Injury}

We further studied the impact of citicoline on the expression of pro-apoptotic and anti-apoptotic factors in HEI-OC-1 cells after neomycin injury. Immunofluorescence staining indicated that the percentage of cleaved caspase 3-positive cells was significantly higher in the neomycin-only group compared with the undamaged controls (Figures $4 \mathbf{A}, \mathbf{B}$ ), but citicolinetreated cells showed a significantly reduced percentage of cleaved caspase 3-positive cells compared to the neomycin-only group (Figures 4A,B). Consistent with these results, we found that the protein levels of cleaved caspase 3 in HEI-OC-1 cells was significantly increased after neomycin treatment compared with the undamaged controls, and the protein levels were significantly reduced by citicoline pretreatment (Figures 4C,D). Furthermore, qRT-PCR analysis showed that the anti-apoptotic factor $\mathrm{Bcl}-2$ was significantly decreased in the neomycin-only group, while the expression of intrinsic and extrinsic pro-apoptotic marker genes, including Bax, Casp3, Casp8, and Casp9, was significantly higher compared to the undamaged controls (Figure $4 \mathrm{E}$ ). In the citicoline-treated group, the expression levels of these intrinsic and extrinsic pro-apoptotic factors were significantly decreased, and expression of the anti-apoptotic factor $\mathrm{Bcl}-2$ was significantly higher compared to the neomycin-only group
(Figure 4E). Together, our results suggested that citicoline is involved in neomycin-induced HEI-OC-1 apoptosis by inhibiting the expression of both intrinsic and extrinsic apoptotic factors.

\section{Citicoline Attenuates Oxidative Stress in Cochlear HCs After Neomycin Injury}

In this experiment, we sought to determine the relationship between citicoline and oxidative stress in cochlear HCs. We dissected and cultured the basilar membranes from P3 mice and treated them with neomycin together with citicoline, and Mito-SOX Red was used to measure mitochondrial ROS levels in the cochleae. Quantification of Mito-SOX Red colocalization with Myo7A showed that the ROS levels were increased in the cochlea after neomycin treatment compared with the undamaged group, and the citicoline-treated cochleae showed significantly lower ROS levels compared to the neomycin-only group (Figures 5A,B).

We next performed qRT-PCR to measure the mRNA expression of redox-related genes in cochlear HCs after neomycin treatment. We found that the expression of the antioxidant genes Gsr, Sod1, and Glrx was significantly decreased in cochlear HCs after neomycin exposure compared with undamaged controls, and the pro-oxidant factor Alox15 was significantly increased in the neomycin-only group (Figure 5C). We then measured the expression of these genes after treatment with citicoline. We found that the expression of the antioxidant genes Gsr, Sod1, and Glrx was significantly increased and the pro-oxidant factor Alox 15 was significantly reduced compared with the neomycinonly group (Figure 5C). 
A
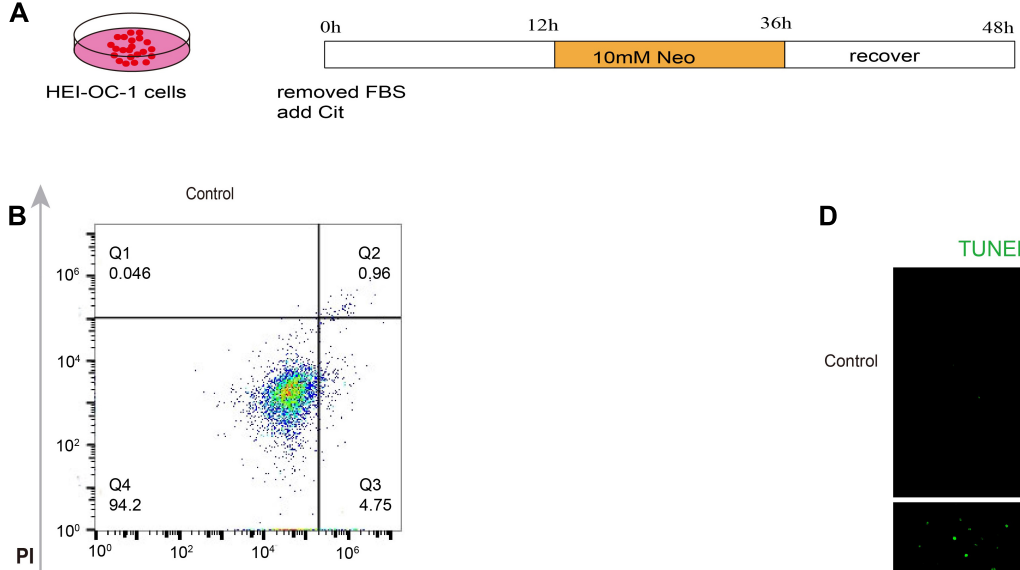

96

D

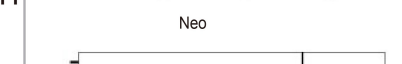

$\mathrm{Neo+Cit}$
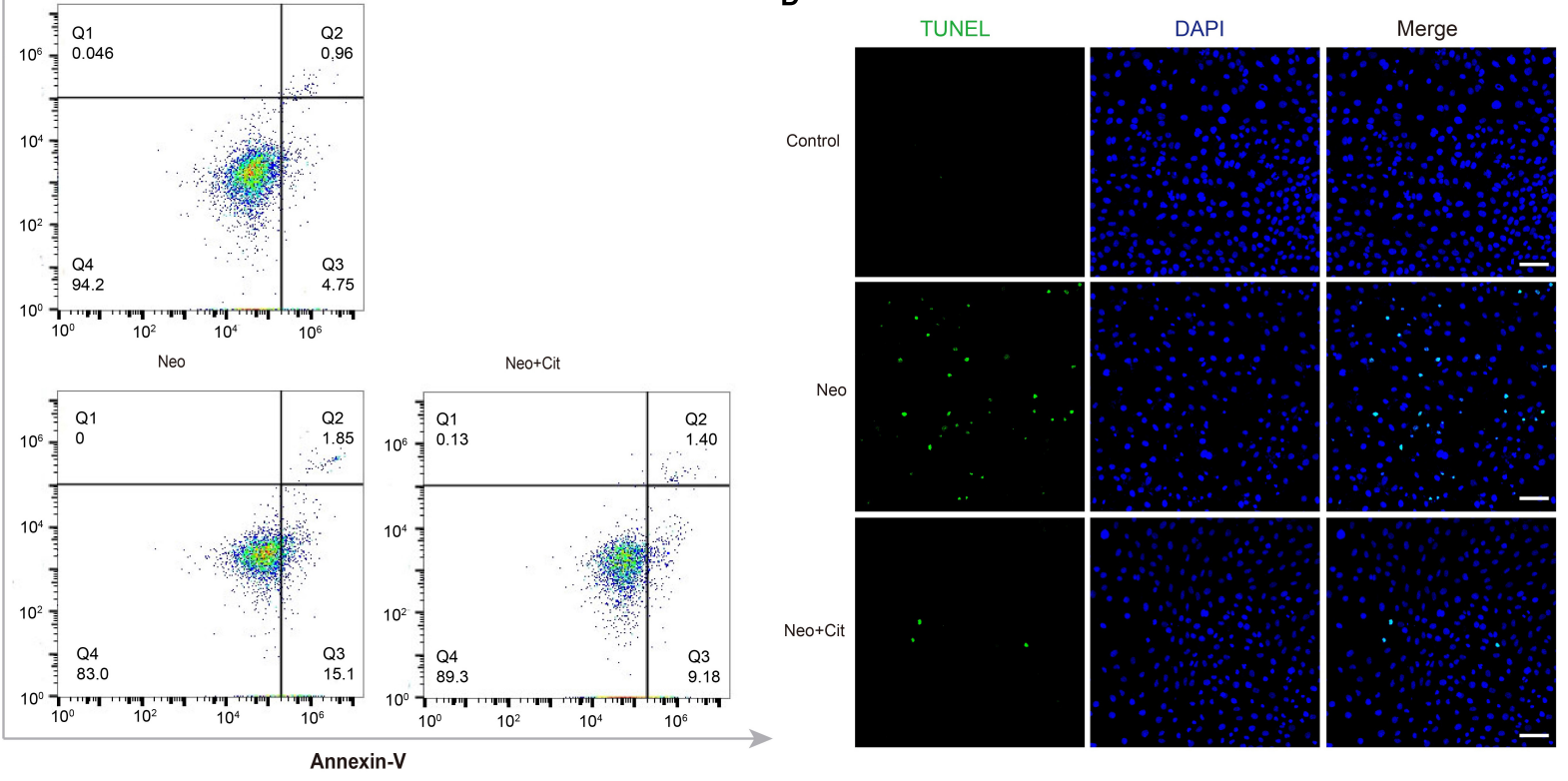

C

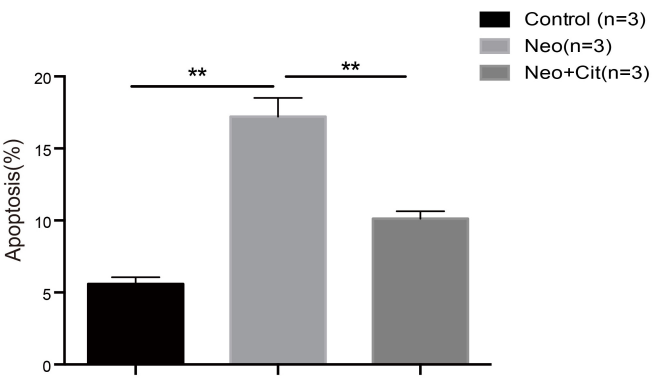

E

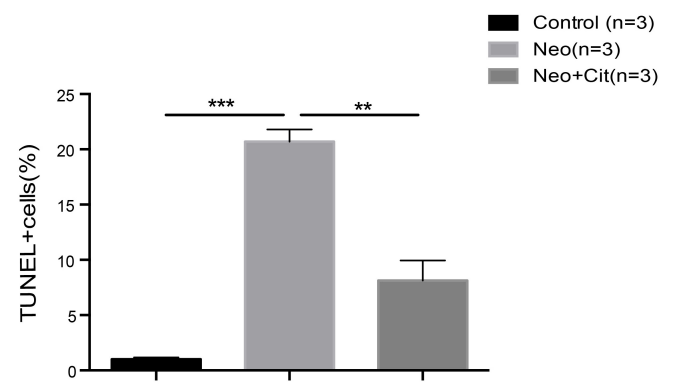

FIGURE 3 | Citicoline protects against apoptosis in HEl-OC-1 cells after neomycin exposure. (A) Schematic diagram of citicoline (Cit) and neomycin addition in cell culture. (B) Apoptosis analysis by flow cytometry after different treatments. The upper right quadrants, lower right quadrants, and lower left quadrants of the images represent late apoptotic cells, early apoptotic cells, and live cells, respectively. (C) Flow cytometry results showing that the percentages of apoptotic cells after neomycin treatment were significantly higher compared with the undamaged controls. The amount of apoptosis induced by neomycin was significantly reduced by pretreatment with citicoline. (D) TUNEL and DAPI double staining showing the apoptotic HEI-OC-1 cells after different treatments. (E) The number of TUNEL/DAPI double-positive cells after neomycin exposure was significantly reduced by treatment with citicoline. Data are shown as mean \pm SD. ${ }^{*} p<0.05,{ }^{* \star} p<0.01$,

\section{Citicoline Increases the Mitochondrial Membrane Potential of HEI-OC-1 Cells After Neomycin Exposure}

To further explore the mechanism behind the role of citicoline in neomycin-induced apoptosis of HEI-OC-1 cells, we used TMRE kits to measure changes in the MMP in HEI-OC-1 cells using immunofluorescence staining and flow cytometry analysis. The TMRE intensity was significantly decreased after neomycin treatment compared to the undamaged control group (Figures 6A-C), and the citicoline-treated cells showed a significantly greater TMRE intensity than the neomycinonly group (Figures $6 \mathrm{~A}-\mathrm{C}$ ). These results demonstrated that citicoline protects HEI-OC-1 cells from apoptosis by inhibiting MMP dysfunction.

\section{Citicoline Inhibits Neomycin-Induced Oxidative Stress in $\mathrm{HEI}-\mathrm{OC}-1$ Cells}

We used Mito-SOX Red to evaluate mitochondrial ROS levels in HEI-OC-1 cells after neomycin treatment. Immunofluorescence and flow cytometry results showed that the ROS levels were 

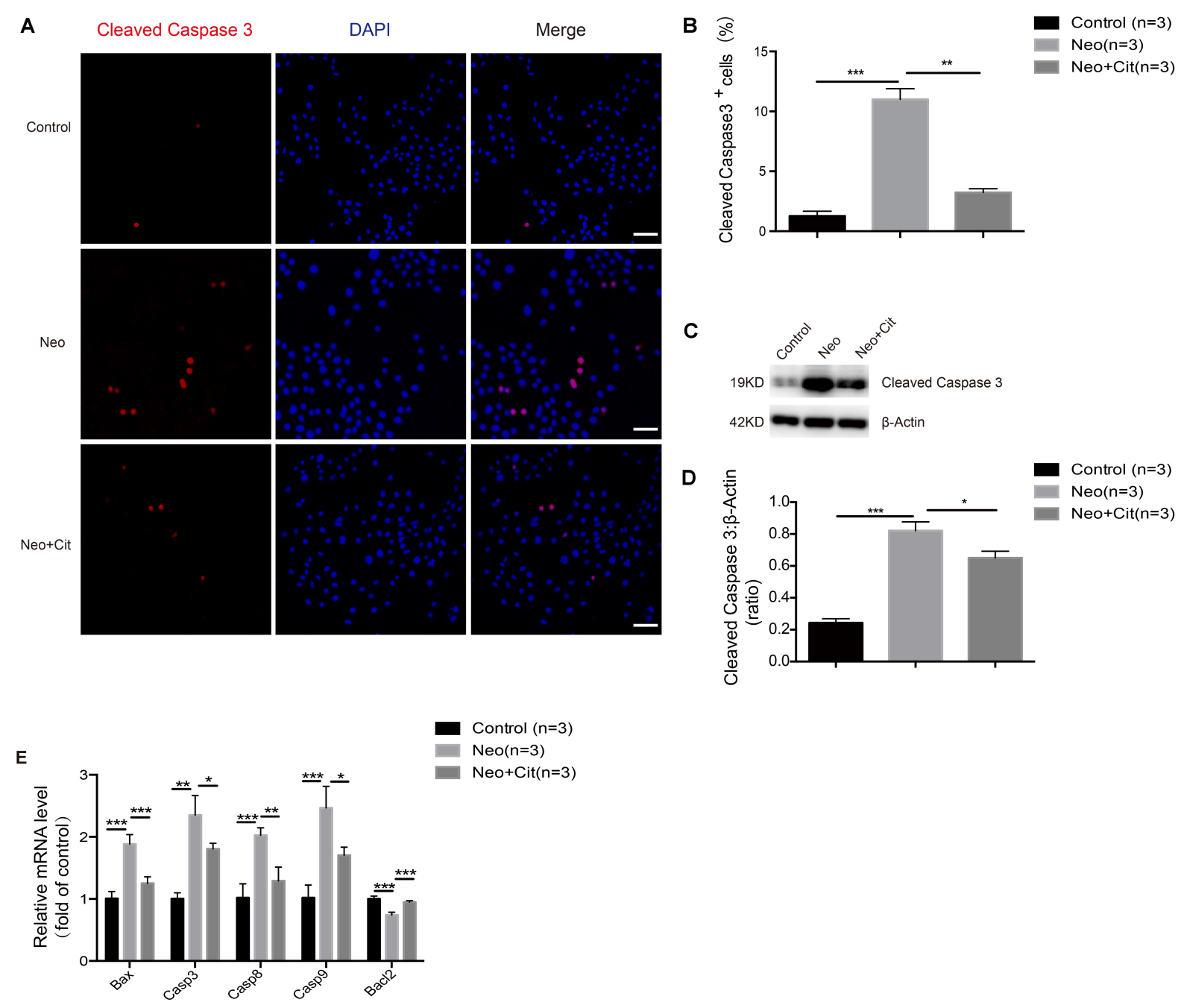

FIGURE 4 | Citicoline reduces the expression of apoptotic factors in HEl-OC-1 cells after neomycin exposure. (A) Cleaved caspase 3 and DAPI double staining confirmed the apoptotic cells after different treatments. (B) Quantification of the numbers of cleaved caspase 3 and DAPI double-positive cells in (A). (C) Western blot showing that the amount of cleaved caspase 3 in the neomycin-only groups was higher than in the undamaged controls. The amount of cleaved caspase 3 induced by neomycin was significantly reduced by pretreatment with citicoline. (D) Quantification of the western blot in (C). (E) The mRNA levels of five apoptosis-related genes were analyzed by qRT-PCR normalized to GAPDH and presented as the fold of control levels. Data are shown as mean \pm SD. ${ }^{\star} P<0.05$, ${ }^{\star \star} p<0.01,{ }^{\star \star \star} p<0.001$. Scale bars $=20 \mu \mathrm{m}$.

significantly increased after neomycin treatment compared with the undamaged group and were significantly reduced in the citicoline-treated group compared with the neomycin-only group (Figures 7A-C).

To further verify our findings, we analyzed the mRNA expression of four redox-related genes by qPCR. The expression of the pro-oxidant factor Alox15 increased after neomycin treatment compared with the undamaged controls, while the antioxidant genes Gsr, Sod1, and Glrx were significantly decreased in the neomycin-only group (Figure 7D). In addition, treatment with citicoline significantly upregulated the expression of the antioxidant genes Gsr, Sod1, and Glrx and reduced the expression of the pro-oxidant factor Alox 15 compared with the neomycin-only group (Figure 7D). Our results indicated that citicoline increased the expression of antioxidant genes and decreased the expression of pro-oxidant genes and thus reduced mitochondrial ROS levels in the cells and prevented apoptosis after neomycin injury.

\section{Citicoline Downregulates the Expression of VDAC1 in HEI-OC-1 Cells After Neomycin Exposure}

VDAC1 is a major channel protein located in the outer mitochondrial membrane, and it plays an important regulatory role in the communication between the mitochondria and other parts of the cell. Compared with the undamaged group, the neomycin-only group was characterized by the obviously enhanced VDAC1-positive staining; however, treatment with citicoline significantly downregulated the mitochondrial 

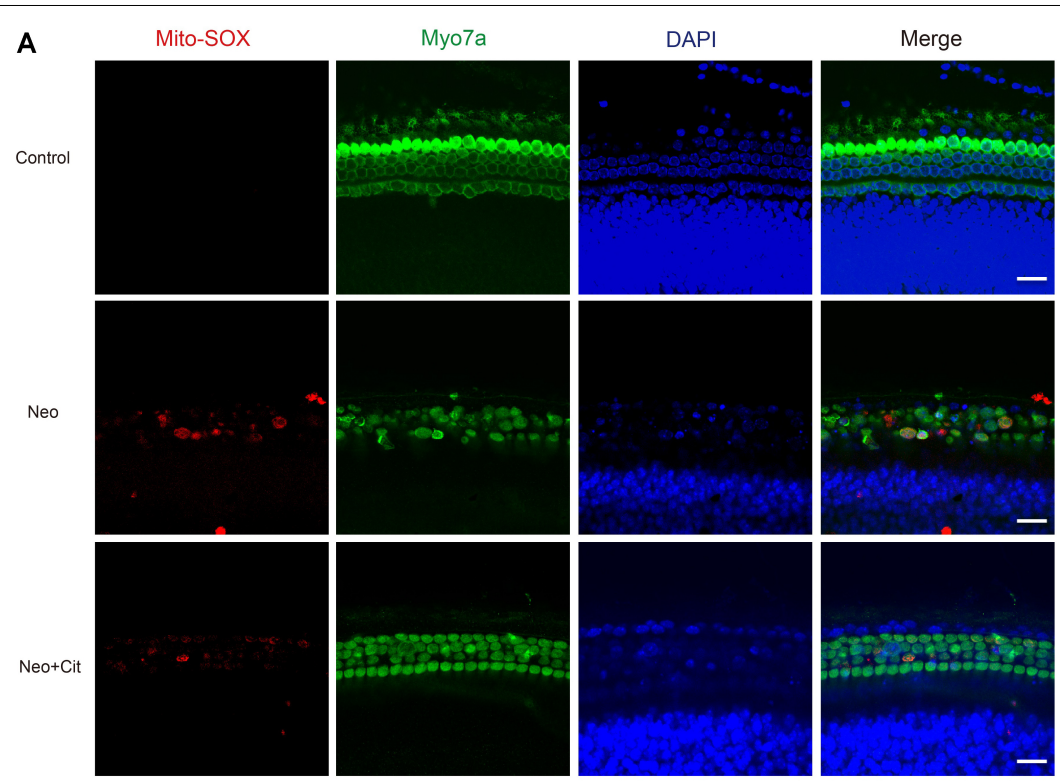

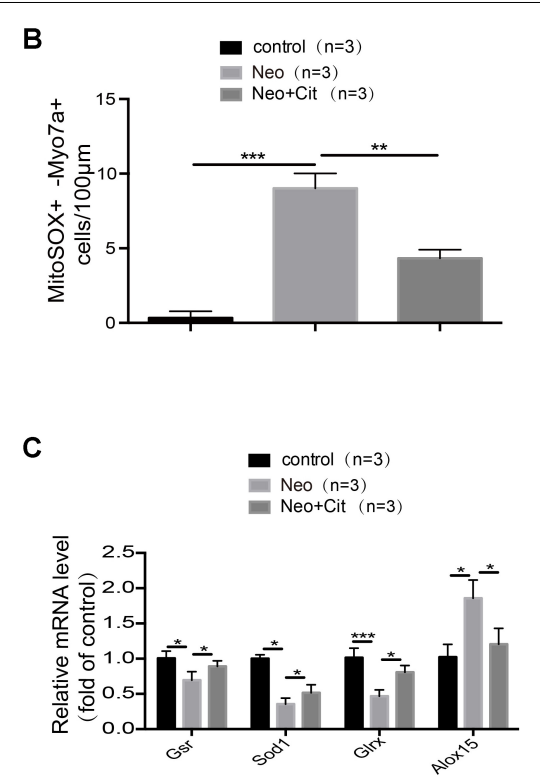

FIGURE 5 | Citicoline attenuates oxidative stress in cochlear HCs after neomycin exposure. (A) Immunofluorescence staining with Mito-SOX Red and anti-Myo7A antibodies in the middle turn of the cochlea after different treatments. (B) Quantification of the numbers of Mito-SOX Red and Myo7A double-positive cells in (A). (C) The mRNA levels of genes related to redox reactions were analyzed by qRT-PCR after neomycin and citicoline treatment and normalized to GAPDH and presented as the fold change compared to control levels. Data are shown as mean $\pm \mathrm{SD} .{ }^{*} p<0.05,{ }^{* \star} p<0.01,{ }^{\star \star *} p<0.001$. Scale bars $=20 \mu \mathrm{m}$.

fluorescence intensity (Figures $\mathbf{8 A}, \mathbf{B}$ ). The VDAC1 staining further confirmed that citicoline inhibits neomycin-induced oxidative stress by reducing mitochondrial ROS levels.

\section{DISCUSSION}

Ototoxic drugs are a common cause of sensorineural hearing loss, and aminoglycosides are the most commonly used ototoxic drugs. Cochlear HCs are susceptible to aminoglycoside-mediated cytotoxicity and cannot be regenerated once damaged, and thus aminoglycoside ototoxicity is usually associated with permanent sensorineural deafness (Nadol, 1993; Lazarou et al., 2015; Correia-Melo et al., 2017). Recent studies have reported that HCs in the mouse cochlea have a very limited ability to regenerate during the neonatal period, but this limited spontaneous $\mathrm{HC}$ regeneration is not sufficient to restore hearing ability once HCs are destroyed by aminoglycosides, and all regenerative ability is lost a few days after birth ( $\mathrm{Li}$ et al., 2016; Ni et al., 2016; Waqas et al., 2016; Lu et al., 2017). To explore the mechanisms through which ototoxic drugs cause auditory sensory cell injury and to take appropriate control measures, it has become especially urgent to prevent and treat such diseases. Previous studies have shown that the toxic effects of aminoglycosides on auditory sensory cells are clearly associated with oxidative stress-induced cellular damage and the induction of apoptosis.

Citicoline, a nucleoside derivative, is an indispensable endogenous intermediate in the biosynthesis of phosphatidylcholine, which has been widely demonstrated to play a therapeutic role in the development of central nervous system injury and neurodegenerative diseases, and citicoline has specific effects on promoting brain function recovery and in promoting wakefulness (Wignall and Brown, 2014). Citicoline is a safe and well-tolerated drug without significant systemic side effects, and it has been shown that $100 \mu \mathrm{M}$ of citicoline is not harmful to retinal cells (Davinelli et al., 2017), and even up to $1000 \mu \mathrm{M}$ citicoline is not harmful to retinal neuroglial cells in vitro (Matteucci et al., 2014). We found that the protective effect of citicoline is dose dependent, and the viability of HEI-OC-1 cells was highest when the concentration of citicoline was $10 \mu \mathrm{M}$. We still observed the protective effect at a concentration of $100 \mu \mathrm{M}$, but the protective effect began to decline significantly when the concentration of citicoline reached $1 \mathrm{mM}$. Taken together, these results confirm that citicoline is safe for HEI-OC1 cells in vitro, which is consistent with the good tolerability profile for citicoline in clinical studies (Supplementary Figure S1).

Citicoline is an old drug, and we often ignore its other functions, such as whether it also protects HCs. Previous studies focusing on nerve cells found that citicoline plays a protective role through anti-oxidation and anti-apoptosis activities (Barrachina et al., 2002; Park et al., 2006), and we hypothesized that citicoline might also have an important protective effect against aminoglycoside-induced HC injury. In this study, we investigated the role of citicoline in both neomycin-induced injury in HEIOC- 1 cells and in cochlear HCs, and we found that citicoline significantly decreased apoptosis after neomycin injury in both contexts (Figures 1, 3).

Apoptosis occurs via both intrinsic and extrinsic pathways (Rybak and Kelly, 2003). The aminoglycosides are believed to induce apoptosis by releasing the apoptotic enzyme activation 

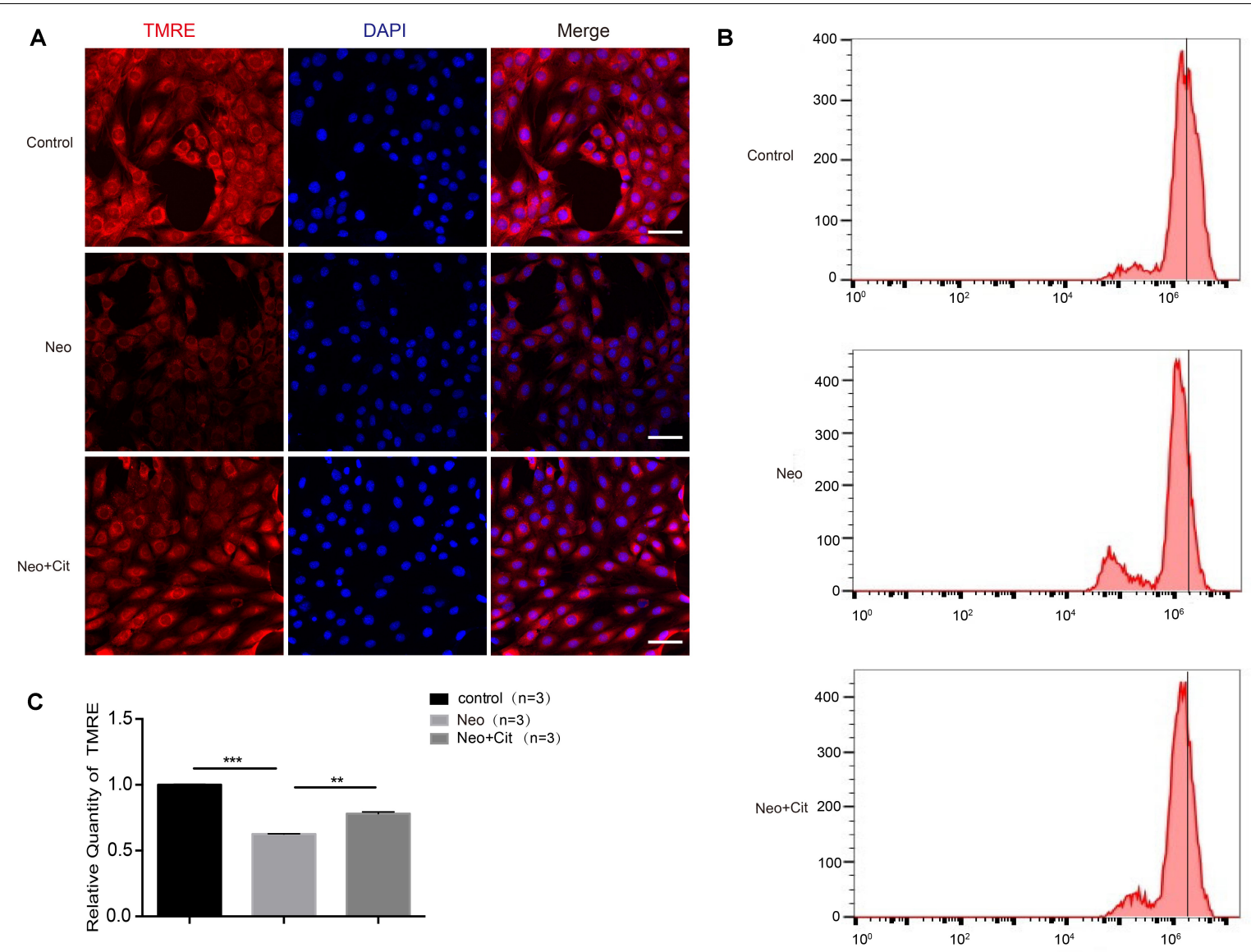

FIGURE 6 | Citicoline increases the MMP of HEI-OC-1 cells after neomycin exposure. (A) The three groups of HEI-OC-1 cells were labeled using the TMRE staining kit. (B) Flow cytometry data confirmed the results in (A). (C) Quantification of the flow cytometry results in (B). The TMRE intensity was significantly decreased after neomycin exposure compared to the undamaged controls. In addition, the TMRE intensity was significantly increased in the citicoline treatment group compared to the neomycin-only group. Data are shown as mean $\pm \mathrm{SD}$. ${ }^{\star} p<0.05,{ }^{\star \star} p<0.01,{ }^{\star \star *} p<0.001$. Scale bars $=20 \mu \mathrm{m}$.

factor that subsequently activates caspases (Karasawa and Steyger, 2011). In this study, we found that cell death and apoptosis dramatically increased in both HEI-OC-1 cells and in cochlear HCs after neomycin injury, while citicoline significantly reduced the neomycin-induced cell death and apoptosis (Figures 2, 4). Furthermore, qRT-PCR analyses indicated that the expression of intrinsic and extrinsic proapoptotic genes (Bax, Casp3, Casp8, and Casp9) was significantly decreased in the citicoline-treated group after neomycin exposure, while the expression of the anti-apoptotic gene $\mathrm{Bcl}-2$ was significantly increased (Figures 2, 4). These results suggest that citicoline plays a critical anti-apoptotic role.

Bcl-2 family proteins regulate the integrity of the mitochondrial outer membrane and play an important role in determining mitochondria-mediated apoptosis. Pro-apoptotic proteins such as Bax translocate to the mitochondrial outer membrane to form oligomeric complexes when they encounter apoptotic signals, resulting in increased mitochondrial outer membrane permeabilization, Cytochrome-C release, and Caspase activation, while anti-apoptotic proteins such as $\mathrm{Bcl}-2$ prevent this process (Martinou and Youle, 2011). Citicoline inhibits apoptosis in nerve cells by promoting the expression of anti-apoptotic factor $\mathrm{Bcl}-2$ and reducing the expression of the pro-apoptotic factor Bax (Levites et al., 2002). In addition, citicoline was also reported to protect the retina by increasing Bcl-2 expression (Schuettauf et al., 2006). Our results show that the expression of the anti-apoptotic gene $B c l-2$ was significantly increased and the expression of the pro-apoptotic gene Bax was significantly decreased after citicoline treatment. This suggested that citicoline can inhibit neomycin-induced HC injury by affecting the expression of Bax and $\mathrm{Bcl}-2$.

Ototoxic drugs cause hearing loss by inducing HC apoptosis, primarily by altering the MMP of the mitochondria (Huang et al., 2000; Wu et al., 2002; Sun et al., 2015; Guan et al., 2016; He et al., 2016; Yu et al., 2017). Mitochondria play an important role in cell metabolism, and aminoglycoside-induced apoptosis is closely related to mitochondrial dysfunction, which leads to decreased MMP and increased ROS (Joza et al., 2001; Chipuk et al., 2004; Coffin et al., 2013; Sun et al., 2014, 2015; Mei et al., 2015). The accumulation of ROS in the mitochondria is an important trigger of apoptosis, and it has been reported that ROS play an important role in noise-induced and ototoxic drug-induced $\mathrm{HC}$ 


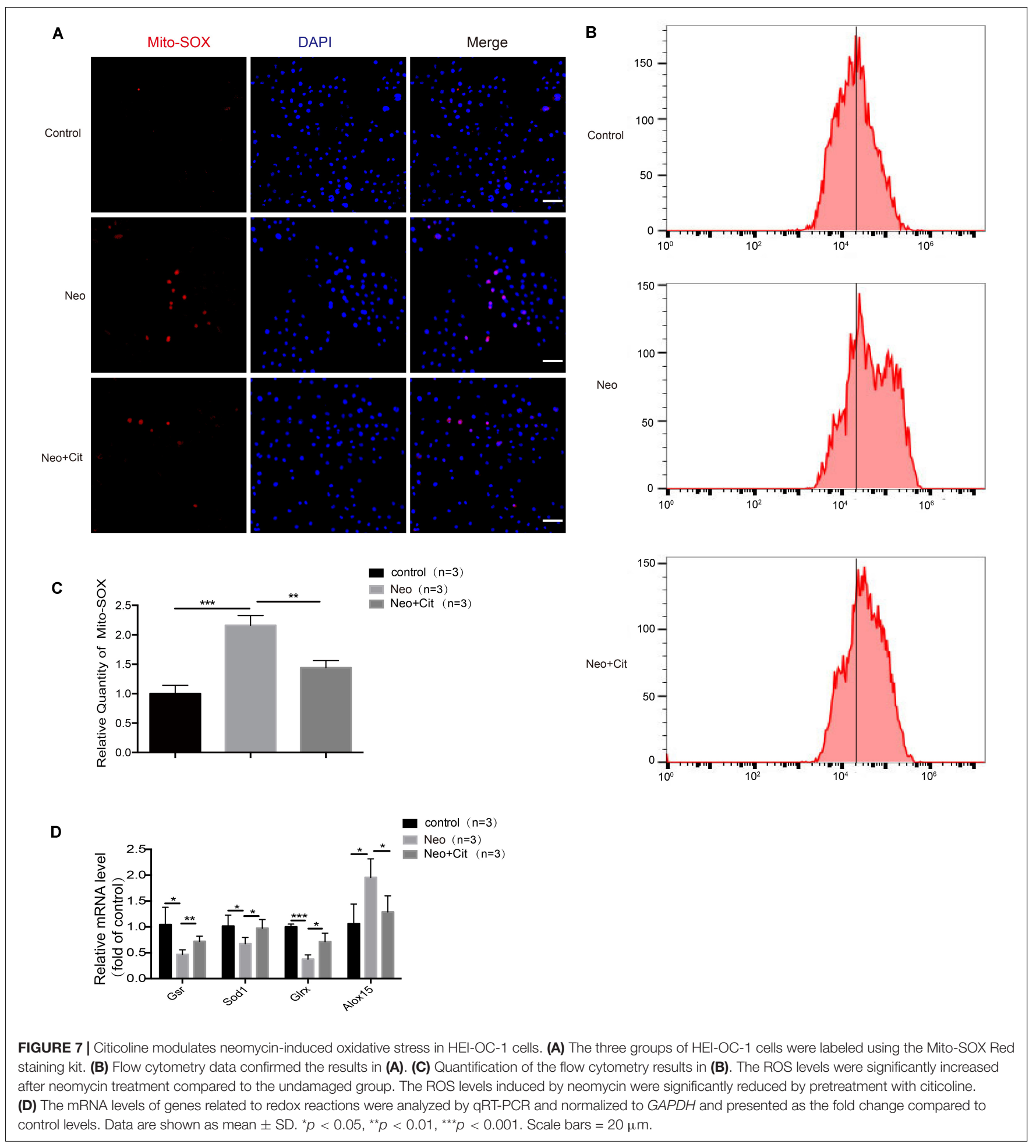

damage and hearing loss (Sun et al., 2014, 2015; Chen et al., 2015). Previous studies have shown that ROS accumulation triggers mitochondrial depolarization, changes mitochondrial membrane permeability, and induces apoptosis (Rybak et al., 2007; Nemec and Khaled, 2008; Chen et al., 2015; Sun et al., 2015). In the present study, we demonstrated that citicoline significantly increased the MMP of HEI-OC-1 cells and decreased ROS levels in HEI-OC-1 cells and in cochlear HCs after neomycin exposure (Figures 5-7), suggesting that citicoline alleviates mitochondrial dysfunction in both cell types after neomycin exposure.

In response to aminoglycoside-induced ROS accumulation, antioxidant genes are upregulated to counteract this 


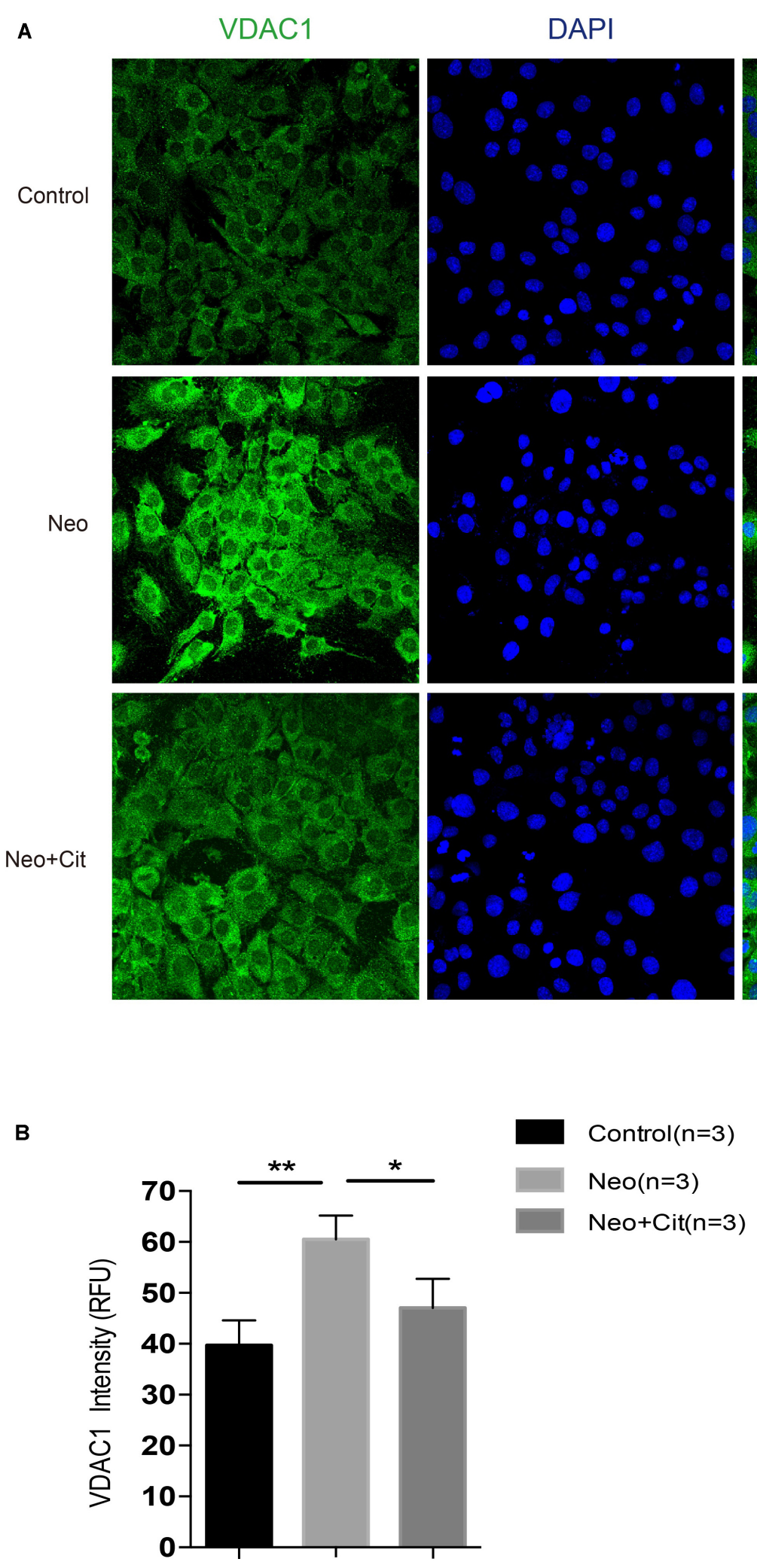

FIGURE 8 | Citicoline downregulates the expression of the VDCA1 of HEI-OC-1 cells after neomycin exposure. (A) The three groups of HEI-OC-1 cells were labeled using the mitochondrial marker VDAC1 and DAPI double staining. (B) Quantitative analysis histogram of VDAC-1 staining intensity scanning in (A). Data are shown as mean \pm SD. ${ }^{*} p<0.05,{ }^{\star \star} p<0.01,{ }^{\star \star *} p<0.001$. Scale bars $=20 \mu \mathrm{m}$. 
accumulation. Therefore, the balance between oxidant and antioxidant gene expression is critical for the rate of ROS accumulation, and many genes coordinate with each other to regulate the balance between the production and scavenging of ROS. Administration of ROS-scavenging antioxidants (Sha and Schacht, 2000; Kawamoto et al., 2004; Kim et al., 2009; Ding et al., 2013), as well as inhibition of oxidase (Kim et al., 2010), can reduce the ROS production, thus attenuating the subsequent $\mathrm{HC}$ death in ototoxic drug-treated cochleae. In this study, the expression of antioxidant genes was significantly decreased and the expression of pro-oxidation factors was significantly increased after neomycin exposure compared with undamaged controls. We also found that citicoline significantly increased the expression of several key antioxidant genes such as Gsr, Sod1, and Glrx and decreased the expression of the pro-oxidation factor Alox15 (Figures 5, 7). Overall, these results indicate that citicoline increases the expression of antioxidant genes, thus leading to decreased ROS levels and preventing neomycin-mediated mitochondrial dysfunction and apoptosis in HEI-OC-1 cells and cochlear HCs.

In our study, the expression of antioxidant genes was significantly downregulated after neomycin exposure. We consider that increased ROS activates the cell defense mechanism in the early stage of cell damage, thus activating the cell antioxidant mechanism and up-regulating antioxidant factors to clear the ROS. However, with the aggravation of cell damage, aminoglycoside exposure induces large increases of ROS in the cochlear HCs that overwhelm the cellular defense mechanisms (Joza et al., 2001; Esterberg et al., 2016). This leads to the imbalance between intracellular oxidation and antioxidation resulting in a significant down-regulation of antioxidant factors and a significant increase in oxidant factors. Because we also previously found that the expression of antioxidant genes decreased after neomycin damaged hair cells (Chen et al., 2015; He et al., 2017), we hypothesize that the downregulation of antioxidant factors is related to the special sensitivity of HCs to aminoglycoside antibiotics, but this still needs further study.

VDAC is a major channel protein located in the outer mitochondrial membrane. It is well recognized that VDAC is involved in many physiological and pathophysiological processes, including $\mathrm{Ca} 2+$ homeostasis (Shoshan-Barmatz et al., 2006, 2010), energy metabolism (Shoshan-Barmatz et al., 2006; Saks et al., 2010), and cell apoptosis (Tsujimoto and Shimizu, 2002; Shoshan-Barmatz et al., 2010). VDAC has three isoforms (VDAC1-3), and VDAC1 is the main isoform mediating cell functions (De Stefani et al., 2012) and plays an important role in regulating intracellular ROS generation and subsequent apoptotic events (Chen et al., 2014). It is necessary for ROS to cross the outer mitochondrial membrane when released from the mitochondria to the cytoplasm, and this process is mediated by VDAC1. Increased intracellular ROS generation can be mostly suppressed by VDAC1 inhibitors, and thus VDAC1 appears to play a dominant role in regulating ROS generation (Chen et al., 2014). In our study, we demonstrated that citicoline significantly downregulated VDAC1 in HEI-OC-1 cells after neomycin exposure (Figure 8), thus leading to decreased ROS levels and inhibition of apoptosis.
Sirtuin 1 (SIRT1), the most conserved member of the NADC-dependent protein deacetylase family, has been shown to have protective effects in various common neurodegenerative disorders (Herskovits and Guarente, 2014). Citicoline has been recently shown to increase SIRT1 protein expression, and this is strongly related to its neuroprotective activities (Hurtado et al., 2013). A previous study showed that increased SIRT1 protects against cisplatin-induced damage to HCs (Xiong et al., 2019). Therefore, we will further study whether the protective effect of citicoline on neomycin-induced HC damage works through SIRT1 activation.

In summary, we first used citicoline on hair cells and clarified the importance of the drug on ear hair cells for the first time. Our study provides the first report that citicoline protects auditory HCs against neomycin injury by preventing mitochondrial dysfunction and the upregulation of antioxidant genes, thus leading to decreased ROS levels and preventing apoptosis. This study therefore provides experimental evidence for the potential clinical application of citicoline to prevent aminoglycoside-induced auditory HC damage.

\section{DATA AVAILABILITY STATEMENT}

The original contributions presented in the study are included in the article/Supplementary Material, further inquiries can be directed to the corresponding author/s.

\section{ETHICS STATEMENT}

The animal study was reviewed and approved by the Animal Care and Use Committee of Southeast University. Written informed consent was obtained from the owners for the participation of their animals in this study.

\section{AUTHOR CONTRIBUTIONS}

ZZ, XF, HL, and JC contributed equally to this work. All the authors made substantial and direct intellectual contributions to the work and approved it for publication.

\section{FUNDING}

This work was supported by grants from the National Key R\&D Program of China (No. 2017YFA0103903), the Strategic Priority Research Program of the Chinese Academy of Sciences (XDA16010303), the National Natural Science Foundation of China (Nos. 81970882, 81900937, 81970884, 81771019, 81822011,81771013 , and 81870721), the Natural Science Foundation from Jiangsu Province (BE2019711), Shenzhen Fundamental Research Program (JCYJ20190814093401920), the K. C. Wong Education Foundation, Boehringer Ingelheim Pharma GmbH, and the Science and Technology Commission of Shanghai Municipality (17ZR1448600 and 18410712400). 


\section{SUPPLEMENTARY MATERIAL}

The Supplementary Material for this article can be found online at: https://www.frontiersin.org/articles/10.3389/fcell.2020.00712/ full\#supplementary-material

FIGURE S1 | Citicoline promotes HEI-OC1 cells survival after neomycin exposure. (A) The CCK-8 kit measured the cell viability after treatment with different neomycin concentrations (1-20 mM) for 24 h. (B) The cell viability was measured

\section{REFERENCES}

Adibhatla, R. M., Hatcher, J. F., and Dempsey, R. J. (2002). Citicoline: neuroprotective mechanisms in cerebral ischemia. J. Neurochem. 80, 12-23. doi: 10.1046/j.0022-3042.2001.00697.x

Barrachina, M., Secades, J., Lozano, R., Gómez-Santos, C., Ambrosio, S., and Ferrer, I. (2002). Citicoline increases glutathione redox ratio and reduces caspase- 3 activation and cell death in staurosporine-treated SH-SY5Y human neuroblastoma cells. Brain Res. 957, 184-190.

Chen, H., Gao, W., Yang, Y., Guo, S., Wang, H., Wang, W., et al. (2014). Inhibition of Vdacl Prevents $\mathrm{Ca}^{2+}$-mediated oxidative stress and apoptosis induced by 5-aminolevulinic acid mediated sonodynamic therapy in Thp-1 macrophages. Apoptosis 19, 1712-1726. doi: 10.1007/s10495-014-1045-5

Chen, Y., Li, L., Ni, W., Zhang, Y., Sun, S., Miao, D., et al. (2015). Bmil regulates auditory hair cell survival by maintaining redox balance. Cell Death Dis. 6:e1605. doi: 10.1038/cddis.2014.549

Chen, Y., Yu, H., Zhang, Y., Li, W., Lu, N., Ni, W., et al. (2013). Cotransfection of Pax2 and Math1 promote in situ cochlear hair cell regeneration after neomycin insult. Sci. Rep. 3:2996.

Chipuk, J. E., Kuwana, T., Bouchier-Hayes, L., Droin, N. M., Newmeyer, D. D., Schuler, M., et al. (2004). Direct activation of bax by p53 mediates mitochondrial membrane permeabilization and apoptosis. Science 303, 10101014. doi: $10.1126 /$ science. 1092734

Choung, Y. H., Taura, A., Pak, K., Choi, S. J., Masuda, M., and Ryan, A. F. (2009). Generation of highly-reactive oxygen species is closely related to hair cell damage in rat organ of Corti treated with gentamicin. Neuroscience 161, 214-226. doi: 10.1016/j.neuroscience.2009.02.085

Clerici, W. J., Hensley, K., DiMartino, D. L., Buttrfield, D. A. (1996). Direct detection of ototoxicant-induced reactive oxygen species generation in cochlear explants. Hear. Res. 98, 116-124. doi: 10.1016/0378-5955(96)00075-5

Coffin, A. B., Rubel, E. W., and Raible, D. W. (2013). Bax, bcl2, and p53 differentially regulate neomycin- and gentamicin-induced hair cell death in the zebrafish lateral line. J. Assoc. ResOtolaryngol. 14, 645-659. doi: 10.1007/ s10162-013-0404-1

Correia-Melo, C., Ichim, G., Tait, S. W. G., and Passos, J. F. (2017). Depletion of mitochondria in mammalian cells through enforced mitophagy. Nat. Protocols 12, 183-194. doi: 10.1038/nprot.2016.159

Cotroneo, A. M., Castagna, A., Putignano, S., Lacava, R., Fantò, F., Monteleone, F., et al. (2013). Effectiveness and safety of citicoline in mild vascular cognitive impairment: the IDEALE study. Clin. Interv. Aging 8, 131-137.

Davinelli, S., Chiosi, F., Di Marco, R., Costagliola, C., and Scapagnini, G. (2017). Cytoprotective effects of citicoline and homotaurine against glutamate and high glucose neurotoxicity in primary cultured retinal cells. Oxidat. Med. Cell. Longev. 2017:2825703.

De Stefani, D., Bononi, A., Romagnoli, A., Messina, A., De Pinto, V., Pinton, P., et al. (2012). VDAC1 selectively transfers apoptotic $\mathrm{Ca} 2+$ signals to mitochondria. Cell Death Differ. 19, 267-273. doi: 10.1038/cdd.2011.92

Ding, D., Qi, W., Yu, D., Jiang, H., Han, C., Kim, M. J., et al. (2013). Addition of exogenous NAD+ prevents mefloquine-induced neuroaxonal and hair cell degeneration through reduction of caspase-3-mediated apoptosis in cochlear organotypic cultures. PLoS One 8:e79817. doi: 10.1371/journal.pone.007 9817

Esterberg, R., Linbo, T., Pickett, S. B., Wu, P., Ou, H. C., Rubel, E. W., et al. (2016). Mitochondrial calcium uptake underlies ROS generation during aminoglycoside-induced hair cell death. J. Clin. Invest. 126, 3556-3566. doi: $10.1172 /$ jci84939 by the CCK-8 kit after treatment with $10 \mathrm{mM}$ neomycin for different times $(0,6,12$, 24, 48 h). (C) The cell viability after neomycin exposure was measured with the CCK-8 kit after pretreatment with different citicoline concentrations (1, 10, $100 \mu \mathrm{M}, 1 \mathrm{mM}, 2 \mathrm{mM}$ ) for different times (6, 12, 24 h). (D) CCK-8 result of $\mathrm{HEI-OC1}$ cells pre-treated with different citicoline concentrations for $6 \mathrm{~h}$ after neomycin exposure. (E) CCK-8 result of HEI-OC1 cells pre-treated with different citicoline concentrations for $12 \mathrm{~h}$ after neomycin exposure. (F) CCK-8 result of $\mathrm{HEI}-\mathrm{OC} 1$ cells pre-treated with different citicoline concentrations for $24 \mathrm{~h}$ after neomycin exposure. Data are shown as mean $\pm \mathrm{SD} .{ }^{*} p<0.05$,

${ }^{\star *} p<0.01,{ }^{* \star *} p<0.001$.

Filomeni, G., De Zio, D., and Cecconi, F. (2015). Oxidative stress and autophagy: the clash between damage and metabolic needs. Cell Death Differ. 22, 377-388. doi: $10.1038 /$ cdd.2014.150

Franco, R., and Cidlowski, J. A. (2009). Apoptosis and glutathione: beyond an antioxidant. Cell Death Differ. 16, 1303-1314. doi: 10.1038/cdd.2009.107

Guan, M., Fang, Q., He, Z., Li, Y., Qian, F., Qian, X., et al. (2016). Inhibition of ARC decreases the survival of HEI-OC-1 cells after neomycin damage in vitro. Oncotarget 7, 66647-66659. doi: 10.18632/oncotarget.11336

He, Y., Yu, H., Cai, C., Shan, S., Chai, R., and Li, H. (2014). Inhibition of H3K4me2 demethylation protects auditory hair cells from neomycin-induced apoptosis. Mol. Neurobiol. 52, 1-10.

He, Z., Guo, L., Shu, Y., Fang, Q., Zhou, H., Liu, Y., et al. (2017). Autophagy protects auditory hair cells against neomycin-induced damage. Autophagy 13, 1884-1904. doi: 10.1080/15548627.2017.1359449

He, Z., Sun, S., Waqas, M., Zhang, X., Qian, F., Cheng, C., et al. (2016). Reduced TRMU expression increases the sensitivity of hair-cell-like HEI-OC-1 cells to neomycin damage in vitro. Sci. Rep. 6:29621.

He, Z. H., Zou, S. Y., Li, M., Liao, F. L., Wu, X., Sun, H. Y., et al. (2020). The nuclear transcription factor FoxG1 affects the sensitivity of mimetic aging hair cells to inflammation by regulating autophagy pathways. Redox Biol. 28:101364. doi: 10.1016/j.redox.2019.101364

Herskovits, A. Z., and Guarente, L. (2014). SIRT1 in neurodevelopment and brain senescence. Neuron 81, 471-483. doi: 10.1016/j.neuron.2014.01.028

Huang, T., Cheng, A. G., Stupak, H., Liu, W., Kim, A., Staecker, H., et al. (2000). Oxidative stress induced apoptosis of cochlear sensory cells: otoprotective strategies. Int. J. Dev. Neurosci. 18, 259-270. doi: 10.1016/s0736-5748(99) 00094-5

Hurtado, O., Hernández-Jiménez, M., Zarruk, J. G., Cuartero, M. I., Ballesteros, I., Camarero, G., et al. (2013). Citicoline (CDP-choline) increases Sirtuin1 expression concomitant to neuroprotection in experimental stroke. J. Neurochem. 126, 816-819.

Joza, N., Susin, S. A., Daugas, E., Stanford, W. L., Cho, S. K., Li, C. Y., et al. (2001). Essential role of the mitochondrial apoptosis-inducing factor in programmed cell death. Nature 410, 549-554. doi: 10.1038/35069004

Kalinec, G. M., Webster, P., Lim, D. J., and Kalinec, F. (2003). A cochlear cell line as an in vitro system for drug ototoxicity screening. Audiol. Neurotol. 8, 177-189. doi: $10.1159 / 000071059$

Karasawa, T., and Steyger, P. S. (2011). Intracellular mechanisms of aminoglycoside-induced cytotoxicity. Integr. Biol. (Camb) 3, 879-886.

Kawamoto, K., Sha, S. H., Minoda, R., Izumikawa, M., Kuriyama, H., Schacht, J., et al. (2004). Antioxidant gene therapy can protect hearing and hair cells from ototoxicity. Mol. Ther. 9, 173-181. doi: 10.1016/j.ymthe.2003.11.020

Kim, H. J., Lee, J. H., Kim, S. J., Oh, G. S., Moon, H. D., Kwon, K. B., et al. (2010). Roles of NADPH oxidases in cisplatin induced reactive oxygen species generation and ototoxicity. J. Neurosci. 30, 3933-3946. doi: 10.1523/jneurosci. 6054-09.2010

Kim, S. J., Park, C., Han, A. L., Youn, M. J., Lee, J. H., Kim, Y., et al. (2009). Ebselen attenuates cisplatin-induced ROS generation through Nrf2 activation in auditory cells. Hear. Res. 251, 70-82. doi: 10.1016/j.heares.2009.03.003

Lazarou, M., Sliter, D. A., Kane, L. A., Sarraf, S. A., Wang, C., Burman, J. L., et al. (2015). The ubiquitin kinase PINK1 recruits autophagy receptors to induce mitophagy. Nature 524, 309-314. doi: 10.1038/nature14893

Levites, Y., Amit, T., Youdim, M. B., and Mandel, S. (2002). Involvement of protein kinase $\mathrm{C}$ activation and cell survival/ cell cycle genes in green tea polyphenol (-)-Epigallocatechin 3-gallate neuroprotective action. J. Biol. Chem. 277, 30574-30580. doi: 10.1074/jbc.m202832200 
Li, W., You, D., Chen, Y., Chai, R., and Li, H. (2016). Regeneration of hair cells in the mammalian vestibular system. Front. Med. 10, 143-151. doi: 10.1007/ s11684-016-0451-1

Liu, L., Chen, Y., Qi, J., Zhang, Y., He, Y., Ni, W., et al. (2016). Wnt activation protects against neomycin-induced hair cell damage in the mouse cochlea. Cell Death Dis. 7:e2136. doi: 10.1038/cddis.2016.35

Lu, X., Shan, S., Qi, J., Li, W., Liu, L., Zhang, Y., et al. (2017). Bmil regulates the proliferation of cochlear supporting cells via the canonical wnt signaling pathway. Mol. Neurobiol. 54, 1326-1339. doi: 10.1007/s12035-016-9686-8

Mangiardi, D. A., Katherine, M. L. W., May, K. E., Messana, E. P., Mountain, D. C., and Cotanche, D. A. (2004). Progression of hair cell ejection and molecular markers of apoptosis in the avian cochlea following gentamicin treatment. J. Comp. Neurol. 475, 1-18. doi: 10.1002/cne.20129

Martinou, J. C., and Youle, R. J. (2011). Mitochondria in apoptosis: Bcl-2 family members and mitochondrial dynamics. Dev. Cell 21, 92-101. doi: 10.1016/j. devcel.2011.06.017

Matsui, J. I., Ogilvie, J. M., and Warchol, M. E. (2002). Inhibition of caspases prevents ototoxic and ongoing hair cell death. J. Neurosci. Official J. Soc. Neurosci. 22, 1218-1227. doi: 10.1523/jneurosci.22-04-01218.2002

Matteucci, A., Varano, M., Gaddini, L., Mallozzi, C., Villa, M., Pricci, F., et al. (2014). Neuroprotective effects of citicoline in in vitro models of retinal neurodegeneration. Int. J. Mol. Sci. 15, 6286-6297. doi: 10.3390/ijms1504 6286

Mei, H., Sun, S., Bai, Y., Chen, Y., Chai, R., and Li, H. (2015). Reduced mtdna copy number increases the sensitivity of tumor cells to chemotherapeutic drugs. Cell Death Dis. 6:e1710. doi: 10.1038/cddis.2015.78

Nadol, J. B. (1993). Hearing loss. N. Engl. J. Med. 329, 1092-1102.

Nemec, K. N., and Khaled, A. R. (2008). Therapeutic modulation of apoptosis: targeting the bcl-2 family at the interface of the mitochondrial membrane. Yonsei Med. J. 49, 689-697.

Ni, W., Zeng, S., Li, W., Chen, Y., Zhang, S., Tang, M., et al. (2016). Wnt activation followed by Notch inhibition promotes mitotic hair cell regeneration in the postnatal mouse cochlea. Oncotarget 7, 66754-66768. doi: 10.18632/oncotarget. 11479

Oshitari, T., Fujimoto, N., and Adachi-Usami, E. (2002). Citicoline has s protective effect on damaged retinal ganglion cells in mouse culture retina. Neuroreport 13, 2109-2111. doi: 10.1097/00001756-200211150-00023

Parisi, V., Coppola, G., Centofanti, M., Oddone, F., Angrisani, A. M., Ziccardi, L., et al. (2008). Evidence of the neuroprotective role of citicoline in glaucoma patients. Prog. Brain Res. 173, 541-554. doi: 10.1016/s0079-6123(08) 01137-0

Park, C. H., Kim, Y. S., Cheon, E. W., Noh, H. S., Cho, C. H., Chung, I. Y., et al. (2006). Action of citicoline on rat retinal expression of extracellular-signalregulated kinase (ERK1/2). Brain Res. 1081, 203-210. doi: 10.1016/j.brainres. 2005.12.128

Prasad, K. N., and Bondy, S. C. (2020). Increased oxidative stress, inflammation, and glutamate: potential preventive and therapeutic targets for hearing disorders. Mech. Ageing Dev. 185:111191. doi: 10.1016/j.mad.2019.111191

Rybak, L. P., and Kelly, T. (2003). Ototoxicity: bioprotective mechanisms. Curr. Opin. Otolaryngol. Head Neck Surg. 11, 328-333. doi: 10.1097/00020840200310000-00004

Rybak, L. P., Whitworth, C. A., Mukherjea, D., and Ramkumar, V. (2007). Mechanisms of cisplatin-induced ototoxicity and prevention. Hear Res. 226, 157-167. doi: 10.1016/j.heares.2006.09.015

Saks, V., Guzun, R., Timohhina, N., Tepp, K., Varikmaa, M., Monge, C., et al. (2010). Structure-function relationships in feedback regulation of energy fluxes in vivo in health and disease: mitochondrial interactosome. Biochim. Biophys. Acta 1797, 678-697. doi: 10.1016/j.bbabio.2010.01.011

Saver, J. L. (2008). Citicoline: update on a promising and widely available agent for neuroprotection and neurorepair. Rev. Neurol. Dis. 5, 167-177.
Schuettauf, F., Rejdak, R., Thaler, S., Bolz, S., Lehaci, C., Mankowska, A., et al. (2006). Citicoline and lithium rescue retinal ganglion cells following partial optic nerve crush in the rat. Exp. Eye Res. 83, 1128-1134. doi: 10.1016/j.exer. 2006.05.021

Secades, J. J., and Lorenzo, J. L. (2006). Citicoline: pharmacological and clinical review. Methods Find Exp. Clin. Pharmacol. 28, 1-56.

Sha, S. H., and Schacht, J. (2000). Antioxidants attenuate gentamicin-induced free radical formation in vitro and ototoxicity in vivo: $\mathrm{D}$-methionine is a potential protectant. Hear Res. 142, 34-40. doi: 10.1016/s0378-5955(00)00003-4

Shoshan-Barmatz, V., De Pinto, V., Zweckstetter, M., Raviv, Z., Keinan, N., and Arbel, N. N. (2010). VDAC, a multi-functional mitochondrial protein regulating cell life and death. Mol. Aspects Med. 31, 227-285. doi: 10.1016/j. mam.2010.03.002

Shoshan-Barmatz, V., Israelson, A., Brdiczka, D., and Sheu, S. S. (2006). The voltage-dependent anion channel (VDAC): function in intracellular signalling, cell life and cell death. Curr. Pharm. Des. 12, 2249-2270. doi: 10.2174/ 138161206777585111

Sun, S., Sun, M., Zhang, Y., Cheng, C., Waqas, M., Yu, H., et al. (2014). In vivo overexpression of $\mathrm{x}$-linked inhibitor of apoptosis protein protects against neomycin-induced hair cell loss in the apical turn of the cochlea during the ototoxic-sensitive period. Front. Cell. Neurosci. 8:248. doi: 10.3389/fncel.2014. 00248

Sun, S., Yu, H., Honglin, M., Ni, W., Zhang, Y., Guo, L., et al. (2015). Inhibition of the activation and recruitment of microglia-like cells protects against neomycin-induced ototoxicity. Mol. Neurobiol. 51, 252-267. doi: 10.1007/ s12035-014-8712-y

Tsujimoto, Y., and Shimizu, S. (2002). The voltage-dependent anionchannel: an essential player in apoptosis. Biochimie 84, 187-193. doi: 10.1016/s03009084(02)01370-6

Waguespack, J. R., and Ricci, A. J. (2005). Aminoglycoside ototoxicity: permeant drugs cause permanent hair cell loss. J. Physiol. 567, 505-521.

Waqas, M., Zhang, S., He, Z., Tang, M., and Chai, R. (2016). Role of Wnt and Notch signaling in regulating hair cell regeneration in the cochlea. Front. Med. 10, 237-249. doi: 10.1007/s11684-016-0464-9

Wignall, N. D., and Brown, E. S. (2014). Citicoline in addictive disorders: a review of the literature. Am. J. Drug Alcohol. Abuse 40, 262-268. doi: 10.3109/ 00952990.2014 .925467

Wu, W. J., Sha, S. H., and Schacht, J. (2002). Recent advances in understanding aminoglycoside ototoxicity and its prevention. Audiol. Neurotol. 7, 171-174. doi: 10.1159/000058305

Xiong, H., Chen, S., Lai, L., Yang, H., Xu, Y., Pang, J., et al. (2019). Modulation of miR-34a/SIRT1 signaling protects cochlear hair cells against oxidative stress and delays age-related hearing loss through coordinated regulation of mitophagy and mitochondrial biogenesis. Neurobiol. Aging 79, 30-42. doi: 10.1016/j. neurobiolaging.2019.03.013

Yu, X., Liu, W., Fan, Z., Qian, F., Zhang, D., Han, Y., et al. (2017). c-Myb knockdown increases the neomycin-induced damage to hair-cell-like HEI-OC1 cells in vitro. Sci. Rep. 7:41094.

Conflict of Interest: The authors declare that the research was conducted in the absence of any commercial or financial relationships that could be construed as a potential conflict of interest.

Copyright (c) 2020 Zhong, Fu, Li, Chen, Wang, Gao, Zhang, Cheng, Zhang, Li, Zhang, Qian, Shu, Chai and Gao. This is an open-access article distributed under the terms of the Creative Commons Attribution License (CC BY). The use, distribution or reproduction in other forums is permitted, provided the original author(s) and the copyright owner(s) are credited and that the original publication in this journal is cited, in accordance with accepted academic practice. No use, distribution or reproduction is permitted which does not comply with these terms. 